\title{
The Unconscious Logic of Decision-making
}

\author{
NELSON MAURO MALDONATO \\ mauro.maldonato@unibas.it \\ University of Basilicata
}

\begin{abstract}
Contemporary science is facing a crucial question: what is the nature of rationality? Over the last 30 years systematic analysis of human thought has come up with new and surprising evidence. In particular, cognitive neuroscience has defined a number of concrete, significant and reproducible cases which are general enough to clarify some regular features of mental processes. Experimental research has shown that in their behaviour the great majority of people follow 'anomalous' spontaneous intuitions, unconsciously adopting adaptive solutions which are incompatible with normative rationality.
\end{abstract}

\section{Introduction}

It is indeed astounding the number of decisions that humans have made from the beginnings of the species until the present day. For one and a half million years, driven by the restlessness that distinguishes them from all other living species, human beings have taken part in a great diaspora which first made them abandon the savannah and then urged them on to the conquest of the most remote and inhospitable corners of the planet: from the edges of deserts to the frozen tundra of the North. Each and every single decision was made by choosing among the possible alternatives in which the future manifested itself to them, often without any possibility of turning back. Moreover, as we are still here to reconstruct human history and analyse the countless decisions made throughout that long and complicated journey across the millennia, those decisions were adaptively successful (Maldonato and Dell'Orco, 2010).

Today we no longer face frequent life-or-death dilemmas, but the number of decisions that we are called upon to make is just as high as it was in the past. We are not even conscious of the majority of these decisions, while others require a great and conscious investment of time and energy. Without human beings' ability to choose, evolution would have been unthinkable or even inconceivable, and in any case it was certainly difficult, for humans had to adapt to the universe on the basis of incomplete, fragmentary information and above all starting with limited cognitive capacities and restricted time limits (Berthoz, 2006). In order to respond to the challenges of the environment, an individual had to first of all be quick: quick in reacting to the attack of a predator and in gaining an escape route, in deciding how to pursue prey, in making use of territory that others were utilizing at that same moment, in the selection of a partner and of a place in which to take refuge, and so forth. But speed on its own would have been insufficient. Decisions of such importance also had to be, if not perfect, at least of sufficient quality to guarantee acceptable results, even though competition with other individuals would inevitably have influenced the level of satisfaction to which a human being could aspire and also the speed with which a solution could be achieved. In 
order to better understand the primitive decision making process, one can imagine an individual looking for food in a territory populated by other individuals. If the number of individuals similar to him/herself was not excessive, he/she could look for food, distinguish the edible food from that which was inedible, and have enough time to eat his/her fill. If, however, that territory was overpopulated, then the old strategies of choice would be completely ineffective, because others would be exhausting the available food supply. The only solution would be to make the quickest decision possible, to be faster than the competitors and to make the best use of the available information. Naturally this second solution would entail advantages but also risks, such as making provisions of inedible food. In any case, the quick decision, even if made on the basis of scarce but fundamentally correct information, would prove to be the winning one (Maldonato, 2010).

For the purposes of survival these factors - correctness of interpretation and speed of decision - are decisive. But in fact the general economy of the mind avails itself of both slowness and rapidity in carrying out the operations essential to survival, such as measuring quantities and distances. Without the use of efficient and effective cognitive strategies which allow for the choosing and the simplifying of problems within brief time limits, our processing of information would not have been (and would not be) successful. Such strategies that reduce the time needed to make decisions have been essential tools for the successful functioning of the cognitive economy of our species. These strategies have, in fact, enabled us to face the limits of our mind (minimizing the effort necessary to make decisions) and to respond to the most pressing internal and external necessities (accumulating rapidly, directly or by inference, the information necessary to make decisions). Therefore, if it is true that evolutionary pressure urged the human mind to accumulate information to provide the basis for making rational decisions, the vast majority of human choices have been made using quick and simple decision strategies. It is probable that these strategies developed as a result of human cognitive limitations (and from their influence on human knowledge of reality), from human drive, and from environmental pressures. The adaptive value of these strategies was revealed in the use of the natural resources supplied by the environment, the strategies proving to be not only remarkably flexible when humans faced new situations, but also ecological, as they tended to result in the suitable use of environmental resources.

\section{Asymmetries of rationality}

It was during the Enlightenment that the controversy over the nature of human rationality came to a head. Over the centuries the term "rational" has had different meanings, indicating reason itself, reasonableness, a domain covering the form and procedures of reason, and a principle of coherence between a system of values and the objectives adopted (Braine, 1978). For those studying the theory of decision making, rationality concerns the choice of the most appropriate means and behaviour for achieving predetermined aims. Rationality is based on the criteria of coherence between thought and action and between means and end; the transitivity that makes it possible to move from the project to its accomplishment by evaluating preferences according to their functionality and optimization so as to achieve the goal through the least possible use of means. This, of course, requires both as much information as possible on the viable alternatives and a certain degree of creativity, above all when there is a significant disparity between the possibilities of information, and its processing, and the complexity of the current situation (Bencivenga, 1985). Reasoning, on the 
other hand, can be defined as a system of procedures: of inference, argumentation, conclusion, induction, deduction, analogy and more besides. In other words, it is the set of mental processes by means of which we draw inferences and elaborate new knowledge on the basis of given knowledge.

Traditionally reasoning has been divided up into two categories: deductive, when one passes from the general to the particular, and inductive, when one passes from the particular to the general. If one considers the nature of the information featuring in the knowledge possessed a priori, one can say that in deductive inferences the conclusions are already implicitly present in the specific premises. This means that no new knowledge is generated; rather, something implicitly known is expressed in a different manner. On the contrary, in inductive inferences the conclusion does not feature in the specific premises, and it reveals something about the reality that was not previously known. It is clear that here, unlike with the deductive inferences, one cannot draw valid conclusions from a logical standpoint (Lemmon, 2008). A further element of classification concerns the nature of the premises. Before drawing an inference the premises may be affirmations which are absolutely certain or with a certain margin of certainty. In the latter case we are dealing with probabilistic reasoning. A probabilistic inference may be either deductive or inductive. In fact there are four categories of non-probabilistic deductive reasoning. In terms of our enquiry the mechanisms of reasoning which underlie decision making are of importance, for this is an activity involving both probabilistic and deductive inferences (Girotto, 1994). The process of logic's "depsychologization", designed to banish ambiguities, imprecisions and individual subjectivity, made a big impact on the first generation of cognitive scientists, convinced that the study of the properties of formal systems could constitute a valid criterion also for investigating the correct functioning of mental mechanisms. Among the various theories of reasoning we shall consider here those of mental logic and mental models, and the heuristics and biases programme.

\section{Uncertainty, risk and adaptive thinking}

Rational thought was long considered to be an activity governed by formal laws designed to maximize expected utility. Yet most of our day to day decisions are based on mental processes which have nothing to do with logic. Everybody will have expressed a judgement or taken a decision trusting in their own gut feelings (Gigerenzer, 2007): I don't trust that person, my sixth sense tells me not to take the risk, I sensed a bargain, and so on. Gigerenzer relates the amusing anecdote of a philosophy professor at Columbia University found racking his brains trying to decide whether to accept an offer from a rival university or stay where he was. A colleague simply said: Just maximize your expected utility - you always write about doing this." Exasperated, the first philosopher responded: "Come on, this is serious" (Gigerenzer and Todd, 1999: 9). Contrary to the received wisdom of economics manuals, it is often gut feelings, emotions or intuition which induce people to make those sudden, spontaneous outbursts - I know what I'll do! or That's my best choice! - which actually manifest judicious rationality. By itself formal logic will never be able to tell us who to marry, who to trust, or even which job to choose; in such cases Plato's charioteer is invariably governed by his emotional horses (Lehrer, 2009).

Intuition is not all that different to the perceptive stratagems we have seen above, a form of instinctive and unconscious knowledge that enables us - instead of calling on logical 
deductive processes - to view and face up to things in a new and often decisive way. Etymologically the term "to intuit" (from the Latin intuèri, to look into) indicates a way of looking or knowing using "the mind's eye": the most natural, ancient, and universal capacity - an authentic biological wisdom - possessed by human beings (Myers, 2002). Intuition comes into play in situations in which temporal and cognitive-computational constraints prevent us reflecting on or evaluating the data at our disposal. It can save us a great deal of hardship, and it is an extraordinary ally when our very survival is at stake. Indeed, this has been the case since the dawn of time. Being able to decipher rapidly the intentions of whoever you are confronting increases your chances of survival (Hogart, 2001). This explains why often the first instants of an encounter can reveal more than hours of conversation.

Besides, in the various cultures the world over, the ability to interpret non-verbal signals is of enormous importance. In reality, the vast majority of human decisions are intuitive, unconscious, and requiring only limited mental input. They enable us to elaborate, rapidly and without any great effort, a considerable amount of information that lies sedimented in our memory, soliciting an immediate and often reliable assessment of the situation on the basis of analogies with past experiences that can reveal unexpected solutions for the problems oppressing us. In any situation in which we have achieved a high degree of experience, an incalculable store of information has been accumulated at the level of "gut instinct". Thus, with just a rapid glance, a chess grandmaster can make the definitive move, or at least the best one possible; an expert entomologist can readily identify the class of the insects she happens to see; or again, a doctor in an emergency situation will recognize an incipient life-threatening risk. In each specific context the ability to distinguish between thousands of different situations and objects is one of the fundamental attributes of the expert and the principal source of intuitions (Simon, 1983).

Over the last twenty years there has been a remarkable surge in the volume of research into the mental mechanisms of instinct, making it difficult, as in other fields, to arrive at a rigorous definition of the term. What is "an intuition": creativity, tacit knowledge, implicit learning and memory, sixth sense, heuristics, emotional intelligence? It really is difficult to say. Intuition has characteristics in common with all these, but also with other definitions. Insight, for example, often considered as synonymous with intuition, regards the sudden comprehension of a problem or a problem-solving strategy, the "Eureka! " moment that arrives after a period of more or less conscious incubation, unblocking the solution to the problem. Intuition takes place almost instantaneously and is made up of a set of emotional and somatic processes, without any role being played (at least apparently) by rational, conscious thought. In fact an intuition almost always has a sensation in the pit of the stomach as its somatic correlate, coming all of a sudden (Maldonato and Dell'Orco, 2011). But what lies behind all this? One stimulating hypothesis (not without metaphysical overtones) is that a crowd of "cognitive workers" are engaged every day in the subterranean regions of our mind, far from the light of consciousness, elaborating extraordinary quantities of information that involve the implicit memory, heuristics, spontaneous inferences, emotions, creativity, and much else besides.

Think, for example, of our ability to intuitively recognize a face. Looking at a photo, our brain breaks down the visual information into subcategories (color, depth, and shape) and simultaneously elaborates each aspect, comparing the reconstructed image with ones stored in memory. And so, immediately and apparently effortlessly, among thousands of different 
faces we recognize a person whom we may not have seen for years. True, there is no comparison with the speed at which a computer can match items: the impulses of biological neurons are much slower than those of silicon neurons. But nonetheless our intuitive and unconscious capacities enable us to carry out an incalculable number of actions: catching a ball, converting the bi-dimensional images of the retina into tridimensional perceptions, doing up our shoelaces, making a move at chess, and an infinity of other things (Kasparov, 2007).

Let us go back for a moment to the situation of driving. We know that when beginners take the wheel, they bring as much attention as possible to the act of driving. They concentrate exclusively on the road, take good care not to talk to other people, and so on. Nonetheless, as time goes on and they acquire experience, the procedures become automatic and their attention is turned to other testing occupations. In reality, things do not always go as they should. How many times, totally occupied by everyday concerns, have we found ourselves at home without any memory of how we got there? And how many times have we missed an exit on the motorway because we were distracted by a phone call or entranced by a song on the radio we had not heard for years? Without a precise directive to a specific destination, we would go on automatically performing the tasks we are used to. It is thanks to the efficiency of this automatic, unconscious activity that we can effortlessly complete the routine matters and concentrate on the important things. Our lives are not regulated merely by conscious choices and actions. Each day we are guided in many of our actions by a sort of automatic pilot. There are days in which, a moment after shutting the front door, we hurriedly check whether we have the keys in our pocket or bag. We do not remember picking them up, even if we did so just a few seconds previously. In the absence of disorders (as in the case of dubitative obsessions) this happens because only the highest cognitive activities attain the level of conscious decisions (Gigerenzer, 2007). Think, for example, of language. Speaking is one of our most important daily actions, and at the same time (apparently) one of the simplest. We utter innumerable word sequences without any effort and perfectly correctly. Just as if, in the engine rooms of our mind, some industrious neuronal workers were intent on composing and decomposing phrases that then come out without the least conscious effort. We do not know how this happens, but it does, and it also happens when we are writing at the computer. The words we see on the screen are the direct expression of the fingers moving over the keyboard, and these in turn are controlled by orders issuing from sophisticated conjunctions of nerves in the midbrain, not from the higher spheres of our mind. In fact, if someone talks to us while we are writing, the fingers do not stop moving, because the indefatigable neuronal workers are capable of terminating the sentence we had begun while we respond to our interlocutor. This phenomenon is all the more surprising in expert pianists, who can quite happily carry on a conversation while their fingers perform a familiar piece. The execution of actions of considerable complexity, like those of someone at the piano, is much more articulated than is suggested by experiments on the programming and performance of simpler movements, contextualized and guided by the executant's skill and judgment. All this was already clear to Lotze in the middle of the nineteenth century:

We see in writing or piano-playing a great number of very complicated movements following quickly one upon the other, the instigative representations of which remained scarcely a second in consciousness, certainly not long enough to awaken any other volition than the general one of resigning one's self without 
reserve to the passing over of representation into action (Lotze, cited in James 1890: 1131).

Without taking into account musical interpretation and the talent of each individual performer, all the components of musical ability derive from a complex interaction between motor learning and temporal elaboration and sequencing, with a crucial role being played by the relations between cortex, cerebellum, and basal ganglia.

\section{Conclusions}

From an evolutionary standpoint the rapidity with which information is gathered has often been (and still is) more important than the precision of our logical inferences. In order to survive, humans have to reason on the basis of fragmentary information, within narrow time limits and restricted cognitive abilities (Gigerenzer, 1997). In the decision-making process the speed of action depends on the use of heuristic and intuitive strategies making it possible to simplify the problems, ensuring efficacy for the cognitive economy of the species. Certainly, when appropriately applied, formal logic can give acceptable results, but its processes are so slow and expensive in cognitive terms as to make it difficult to use. This surely explains why, in general, we are so inefficient at making calculations and so brilliant at estimating quantities, distances and the other variables required for survival. Moreover a lot of experimental evidence from cognitive psychology has confuted the classical hypothesis of an innate logico-formal competence, showing how the use of heuristics and intuitive decisionmaking strategies is part and parcel of human rationality (Maldonato, 2010). For this reason a realistic attempt to understand our logical capabilities requires a broad notion of logic involving the cognitive processes without confining itself to computational processes. Above all it has to recognise the component elements of fallibility. Rationality is an extremely complex ideal which we can only approach by successive approximations, as if it were almost out of our reach. Rational choices, judgements and behaviour are not an ideal extension of spontaneous psychological norms. Obviously this does not imply the existence of a conflict between spontaneous and ideal rationality. These two aspects are neither in harmony nor in conflict with each other because reason is not a faculty of our species which acts spontaneously and without effort. If it is true that rationality is conditioned by severe evolutionary limitations, the difficulty in deciding and facing up to uncertainty is not only linked to the inadequacy of the architecture of our minds but also to an 'external' model of uncertainty which does not correspond to the way in which our mind naturally functions (Shafer and Pearl, 1990).

New conceptual paradigms and new programmes for experimental research are called for in order to redefine the role of external restrictions on human action (concerning resources and available information) and reinstate the importance of the internal restrictions (limitations on calculation ability, on the capacity of memory and so on). All this should be contemplated in a more general theoretical framework - natural logic - based not on metaphysical assumptions but on the concrete evidence provided by cognitive neurosciences. It is surely not far-fetched to imagine individuals adopting rules of behaviour enabling them on one hand to simplify their own decision-making models and on the other to coordinate with other agents so as to reduce the degree of uncertainty which characterises any complex system. 